\title{
Focus Variation - a Robust Technology for High Resolution Optical 3D Surface Metrology
}

\author{
Reinhard Danzl* - Franz Helmli - Stefan Scherer \\ Alicona, Grambach, Austria
}

This article describes and evaluates the focus variation method, an optical $3 D$ measurement technique. The goal is to analyse the performance of the method on a series of typical measurement tasks including roughness measurements, form and wear measurements. First, a comparison of roughness measurements between the proposed method and a tactile device on a newly developed roughness standard is made.

Results show that both systems deliver $R_{a}$ values that are comparable to each other with differences of a few nanometers. Afterwards form measurements are performed on a calibration standard with hemi-spherical calottes, showing a repeatability of sphere measurements $<100 \mathrm{~nm}$. Finally, two typical engineering applications are provided. The first is wear measurement of cutting tools, the second the inspection and classification of welding spots. Both applications demonstrate the ability of the method to measure steep surface flanks up to $80^{\circ}$ and surfaces with difficult reflectance behaviour.

(C)2011 Journal of Mechanical Engineering. All rights reserved.

Keywords: metrology, 3D, optical, focus variation, measurement, roughness, form, accuracy, comparison, tactile

\section{INTRODUCTION}

The 3D measurement of technical surfaces is a crucial part in checking and controlling the properties and the function of materials or engineering parts. Traditionally, 3D measurements have been performed merely by tactile devices, which can be divided into two main categories. Among the first are contact stylus systems for the measurement of small scale surface features such as surface roughness.

These systems typically operate with a stylus tip, which is traced along a profile over the specimen surface in order to deliver roughness parameters such as $R_{a}, R_{q}$, and $R_{z}$. Among the second category are (micro) coordinate measurement machines (CMMs) where a stylus tip, usually a synthetic ruby ball, is moved to (few) different positions on the specimen in order to measure large scale features such as different form parameters (e.g. a sphere radius, the cylinder diameter, etc.). A good overview of surface metrology systems in general and tactile devices in particular can be found in [1]. Tactile systems have a long tradition in surface measurement and are well understood and accepted in science and industry. Moreover, a lot of international standards on tactile systems exist, which describe the basis structure of a tactile system [2] and standards how to calibrate it [3].

Nevertheless, optical measurement devices have become increasingly popular in the last decade as described by Jiang [4] in his historical overview of surface metrology. This is above all due to their ability to perform area based measurements which are a prerequisite for many powerful surface texture parameters [5]. Although tactile systems nowadays are also able to perform area based measurements, such measurements usually last very long. Apart from the measurement time, there is a range of additional advantages of optical instruments. Firstly, they operate in a noncontact way and therefore, do not damage the surface. Secondly, they usually do not require as much maintenance as a tactile instrument since there are typically none or only very few parts that have to be regularly replaced. Moreover, they do not suffer from several limitations of tactile systems such as a "smoothing effect" of surface profiles due to the radius of the contact stylus tip.

In the field of optical measurement many technologies have become increasingly popular recently. Among them are methods based on white light interferometry, phase shifting

*Corr. Author's Address: R\&D Team, Alicona, Teslastraße 8, 8074 Grambach, Austria, 
interferometry, confocal microscopy, chromatic probe microscopy, structured light techniques, atomic force microscopy and scanning electron microscopy [6]. However, also many optical techniques have their limitations when measuring certain surfaces. Optical techniques that have been typically used, such as white light interferometry are very good for measuring smooth surfaces, but have restrictions in terms of complex geometry measurement, large $\mathrm{Z}$ heights, high slope angles and high aspect ratio measurements. White light interferometry for example, has been found to produce erroneous results for roughness measurements of periodic standards with $R_{a}$ values between 50 and $300 \mathrm{~nm}$ [7].

Another report shows jumps or spikes of half the mean wavelength, which is reported more frequently as the surface gradient increases or when there is a step discontinuity [8]. Other typical limitations of optical instruments are summarized in [1], where one of the most important ones is the limitation of the maximum measurable flank angle in relation to the numerical aperture of the used objective. For objectives with low numerical aperture light can only be gathered by an instrument if the surface topography gradients are sufficiently small, otherwise no information can be obtained. Another limitation is the lateral resolution which is typically limited by the wavelength of visible light (> $400 \mathrm{~nm}$ ).

Here, we present and evaluate the technology focus variation (FV), a rather new technique which exploits the small depth of focus of an optical system with vertical scanning to provide topographical and colour information from the variation of focus. In contrast to other optical techniques, two issues should be especially adressed. First, the method is not limited to coaxial illumination or other special illumination techniques, which allows to overcome some limitations with respect to the maximum measurable slope angle. Second, the technology delivers true colour information for each measurement point. This article will evaluate the performance of the technology with respect to the measurement of small scale surface roughness, the measurement of form and the measurement of steep surface flanks.

There are many studies on the evaluation of optical surface instruments. Typically, such an evaluation is based on the measurement of special parameters of a surface (e.g. roughness, form elements) which are compared to measurements performed by a certified measurement institute. The difficulty hereby is that most reference measurements are performed by tactile devices and that the used standards are especially designed for tactile devices, which makes a comparison difficult. This is e.g. addressed by Dietsch [9] who has compared two tactile and two optical devices (WLI and confocal) using roughness standards and step height artefacts. He concludes that the results do not always correlate which may be among others due to the fact that the standards are not suitable for optical measurements.

There are several publications where the focus variation method has been evaluated. In [10] an instrument based on focus variation and other instruments have been used for form measurements on cylindrical parts of a micro contour artefact. The measurements of the FV instrument lead to radius deviations $<200$ to 300 $\mathrm{nm}$ on radius measurements of the cylindrical elements. In [11], a comparison of FV has been made using a random roughness standard leading to roughness measurement results of the system that lay within the uncertainty range of the tactile measurement.

Apart from evaluations with calibration standards several articles have been published on the use of focus variation instruments for special applications. In [12] for example a FV instrument has been used for the measurement of dental erosion and has been compared to tactile measurements. Both technologies showed similar trends and focus variation has been found to be a suitable tool for the proposed task without the disadvantage of the tactile device making visible scratch marks.

Another study [13] compares focus variation to traditional indirect measurement methods for the roughness of paper, which are based on measuring air flows. The results show that a discrimination of papers with different roughness is also possible with the FV method and not only by traditional indirect methods.

In this article an extension to previous studies is given by providing both, evaluations on different calibration targets and on typical 
engineering examples as shown in the next section.

\section{EVALUATION OF FOCUS VARIATION}

First, a description of the focus variation principle and the focus variation instrument that is used for the evaluations in this section is given. Afterwards several evaluations of the instrument are provided; two of them based on special calibration standards. The first application is the roughness measurement of newly developed roughness standards with sinusoidal profiles (Section 1.2). Hereby, the focus is particularly on the comparison of the results between the device and a traditional tactile system. In the second application (Section 1.3) the focus is on form measurements, in particular the measurement of hemi-spherical calottes that are part of a special calibration target. The remaining two sections show two typical applications. The first is the wear measurement of milling-cutters (Section 1.4) where, in particular, the possibility to measure steep flanks is evaluated. Finally, a typical engineering example is provided, that is the measurement and inspection of welding spots (Section 1.5), which is challenging due to the difficult reflectance characteristics of welding spots.

\subsection{D Measurement with Focus Variation}

Focus variation [14] combines the small depth of focus of an optical system with vertical scanning to provide topographical and colour information from the variation of focus. In the following, the operating principle is described based on a schematic system shown in Fig. 1. The main component of the system is a precision optic containing various lens systems that can be equipped with different objectives, allowing measurements with different resolution. With a beam splitting mirror, light emerging from a white light source is inserted into the optical path of the system and focused onto the specimen via the objective. Depending on the topography of the specimen, the light is reflected into several directions as soon as it hits the specimen via the objective. If the topography shows diffuse reflective properties, the light is reflected equally strong into each direction. In case of specular reflections, the light is scattered mainly into one direction.

All rays emerging from the specimen and hitting the objective lens are bundled in the optics and gathered by a light sensitive sensor behind the beam splitting mirror. Due to the small depth of field of the optics only small regions of the object are sharply imaged. To perform a complete detection of the surface with full depth of field, the precision optic is moved vertically along the optical axis, while continuously capturing data from the surface. This means that each region of the object is sharply focused. Algorithms convert the acquired sensor data into 3D information and a true colour image with full depth of field. This is achieved by analyzing the variation of focus along the vertical axis.

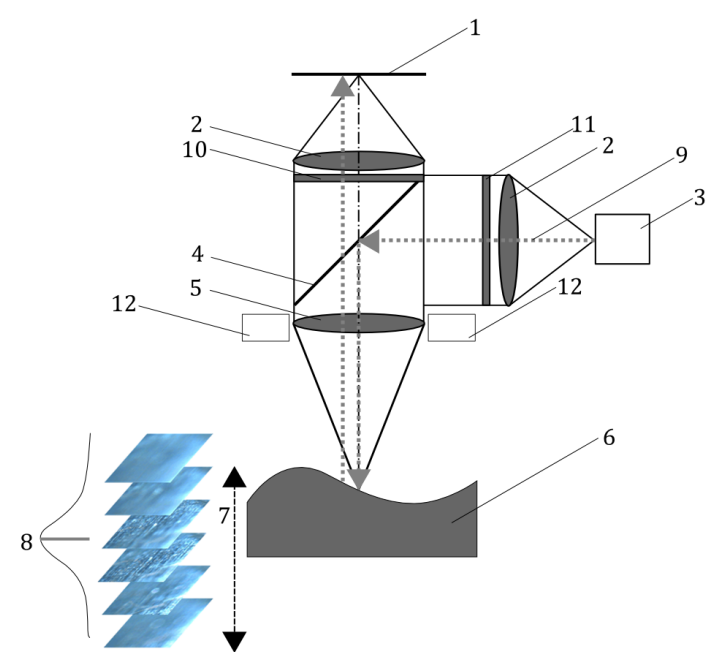

1. array detector, 2. lenses, 3. white light source, 4. beam splitter, 5. objective, 6. specimen, 7. vertical scan, 8. focus curve, 9. light beam, 10. analyzer, 11. polarizer, 12. ring light

Fig. 1. Schematic diagram of a typical measurement device based on focus variation

In this article the focus variation instrument InfiniteFocus is evaluated. Its vertical resolution depends on the chosen objective and can be as low as $10 \mathrm{~nm}$. The vertical scan range depends on the working distance of the objective and ranges from 3.2 to $22 \mathrm{~mm}$. The $x-y$ range is determined by the used objective and typically ranges from $0.14 \times 0.1$ to $5 \times 4 \mathrm{~mm}$ for a single measurement. 
By using special algorithms and a motorized $x-y$ stage the $\mathrm{x}-\mathrm{y}$ range can be exceeded up to $100 \mathrm{x}$ $100 \mathrm{~mm}$ and more.

In contrast to many other optical techniques that are limited to coaxial illumination, the maximum measurable slope angle is not only dependent on the numerical aperture of the objective. However, many different illumination sources (such as a ring light), which allow the measurement of slope angles exceeding $80^{\circ}$ are possible. Since the technique is very flexible in terms of using light, most limitations when measuring surfaces with strongly varying reflection properties within the same field of view can be avoided.

In addition to the scanned height data, focus variation delivers a colour image with full depth of field which is registered to the $3 \mathrm{D}$ points. This provides an optical colour image which eases measurements as far as the identification and localization of measurement fields or distinctive surface features are concerned. Since the described technique relies on analyzing the variation of focus, it is only applicable to surfaces where the focus varies sufficiently during the vertical scanning process. Surfaces not fulfilling this requirement such as transparent specimen or components with only a small local roughness are hardly measurable. Typically, focus variation delivers repeatable measurement results for surfaces with a local $R_{a}$ of $10 \mathrm{~nm}$ at a cut-off wavelength $L_{c}$ of $2 \mu \mathrm{m}$.

\subsection{Roughness Measurement on a Newly Developed Roughness Standard}

The measurement of surface roughness is one of the most common and important ways to judge the quality of a technical surface. In order to verify whether a metrology device is able to measure certain types of roughness accurately, various roughness standards with calibrated roughness values are available.

Below, the process of performing a roughness measurement comparison between the focus variation instrument InfiniteFocus and a tactile device on a newly developed roughness standard is described.

After a review of the main problems that may occur when making such a comparison, the roughness standard and the results of the comparison are described.

The main problem of contact stylus instruments in the context of roughness measurement is that the form of the contact stylus tip has a smoothing effect on the surface profile and can therefore influence the measurement result. Another error source of contact stylus instruments is that the stylus tip may modify the surface if the material is not hard enough (Fig. $3 b)$. Sometimes the stylus tip is not traced along a straight line but may be deflected, e.g. when composite materials consisting of very smooth and very hard components are measured.

a)

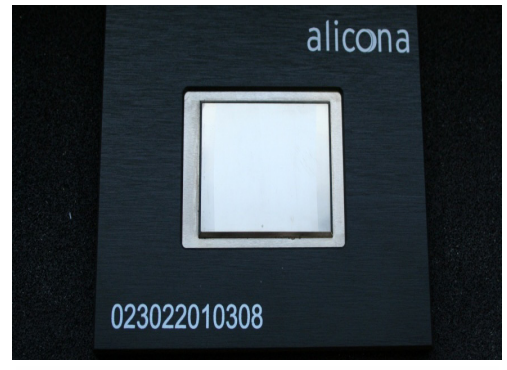

$\mathrm{Sm}$

b)

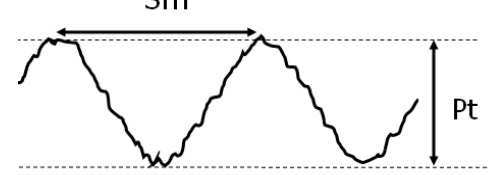

Fig. 2. Periodic roughness standard used for the comparison between a focus variation instrument and a tactile system, a) photograph of the standard, b) schematic height profile of the roughness standard showing the sinusoidal structure, the nano-roughness and the meaning of the parameters $S m$ and $P t$

The main problem of optical instruments is that most existing roughness standards are rather smooth and can hardly be measured with several optical instruments. Therefore, a new roughness standard which contains a certain amount of nanoscale roughness and which is well-measurable with optical devices, has been developed.

The roughness standard used for the comparison is a precision roughness specimen [15] with a regular periodic sinusoidal profile with a nominal peak-to-peak spacing $S m=50 \mu \mathrm{m}$, a peak-to-valley height $P t=1.5 \mu \mathrm{m}$ and a resulting nominal $R_{a}=0.5 \mu \mathrm{m}$. 


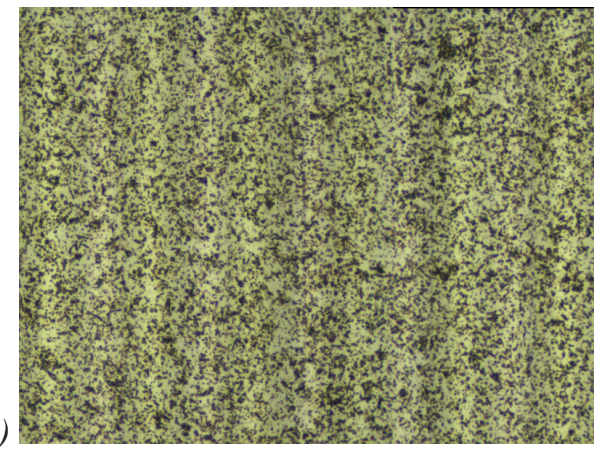

b)

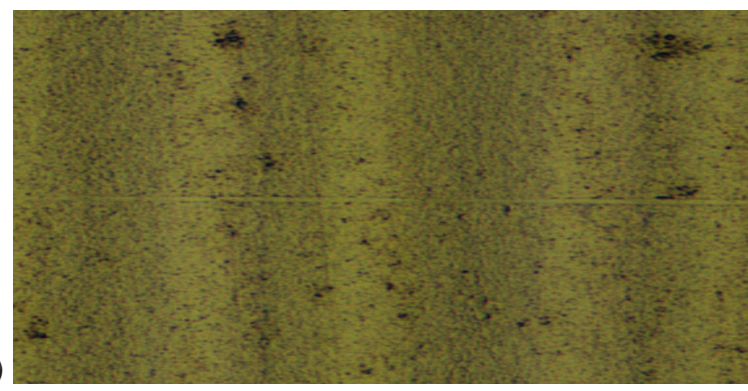

c)
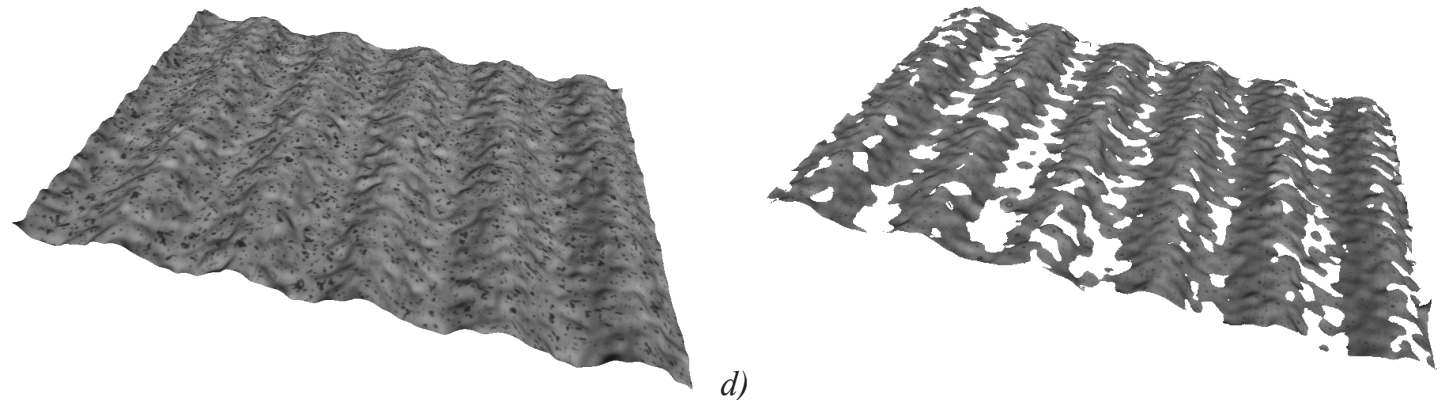

d)
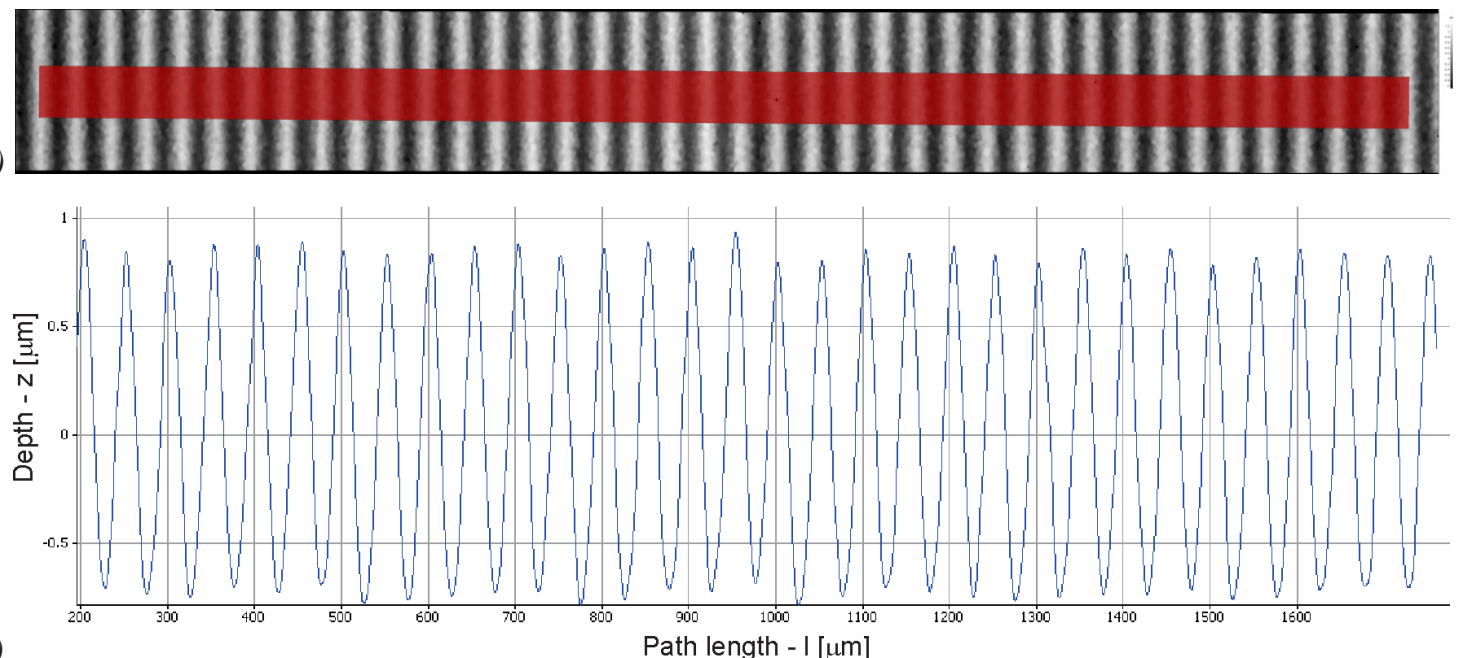

Fig. 3. 3D measurement of a periodic roughness standard $\left(R_{a}=500 \mathrm{~nm}\right)$ with focus variation, a) sharp color image of the roughness standard provided by focus variation, $b)$ detailed view of a sharp

colour image show"sing the horizontal trace of a contact stylus instrument that has been used for reference roughness measurements, c) 3D measurement of the newly developed roughness standard with superimposed nano-roughness, d) 3D measurement of a conventional roughness standard without nanoroughness, e) height map of the roughness standard with profile path and f) surface profile obtained from the profile path in e)

Starting from the precision diamondturned master specimen, various electroformed nickel replicas, which are all faithful copies of the original and of each other, were produced.
The sloping flanks between the peaks and valleys of the sinusoidal roughness profile, however, are smooth and shiny; and in order to introduce some nano-roughness onto these surfaces, the nickel 


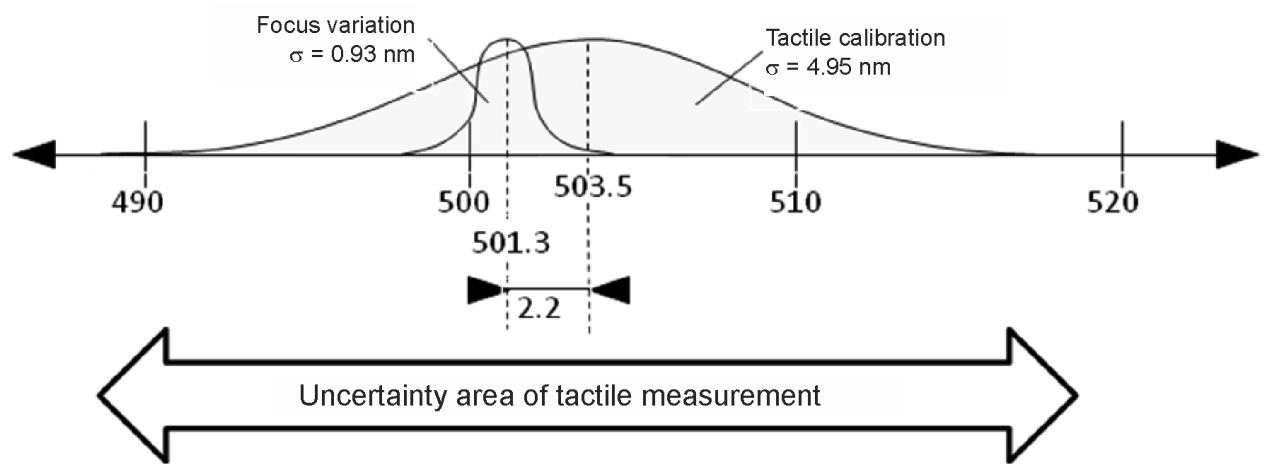

Fig. 4. Visualisation of the roughness measurements by focus variation and the tactile instrument showing the mean $R_{a}$ values and the standard deviation $\sigma$ of the measurements (all values are in $\mathrm{nm}$ )

specimens were etched with a dilute acid solution for varying lengths of time. The roughness standard is shown in Fig. 2a whereas Fig. 2b shows a schematic height profile of the standard and the meaning of the parameters $S m$ and $P t$. The latter diagram illustrates both the overall sinusoidal shape formed by the machining process, and also the superimposed random nano-roughness, which is the result of the acid etch. Note that the nanoroughness is relatively small in comparison to the sinusoidal roughness, so that the overall $R_{a}$ values measured on the etched surface will be very close (within $\sim 1 \%$ ) to those measured on the unetched sinusoidal surface.

Table 1. Comparison of the measurements performed by the tactile instrument and by the device based on focus variation

\begin{tabular}{|l|c|c|}
\hline & $\begin{array}{c}\text { Tactile } \\
\text { instrument }\end{array}$ & $\begin{array}{c}\text { Focus } \\
\text { variation }\end{array}$ \\
\hline \# Measurements & 30 & 25 \\
\hline Mean $R_{a}$ & $503.5 \mathrm{~nm}$ & $501.32 \mathrm{~nm}$ \\
\hline $\operatorname{Std} R_{a}$ & $4.95 \mathrm{~nm}$ & $0.93 \mathrm{~nm}$ \\
\hline
\end{tabular}

A 3D measurement of the standard is provided in Fig. 3c together with a sharp measured image in Fig. 3a. In order to calculate the roughness of the surface, a surface profile has been extracted along a horizontal profile path as shown in Fig. 3e. The measured surface profile is visualized in Fig. 3f showing the regular sinusoidal shape of the surface. The need for the superimposed nano-roughness is visualized in
Fig. 3d where the measurement of a traditional roughness standard without nano-roughness is shown. The measurement is not complete in comparison to those of the newly developed standard in Fig. 3c, since the traditional standard does not have enough small scale structures that can be exploited by the focus variation method.

In order to compare the roughness measurements of the tactile and the optical system, the following procedure has been used. First, the roughness standard has been measured by the tactile instrument at 30 different positions arranged in three straight rows of ten each. This coverage of the whole measuring area serves as a check upon the uniformity of the roughness values from place to place. For the FV device only a single measurement position has been used, where roughness measurements have been repeated 25 times. This allowed a comparison of the measurement results to those of the tactile system and to calculate the repeatability of the measurements of the optical system.

The results of this measurement row are graphically visualized in Fig. 4, showing the different measurement results as well as a Gaussian distribution curve depending on the repeatability of the measurements. It should be noted that the measurements of the tactile instrument have been performed at 30 different positions, so that the standard deviation contains the variability of the measurement device and the variability of the roughness standard. In contrast to this, the measurements by focus variation have all been performed at the same position. As a 
result, the standard deviation of the focus variation instrument only contains the variability of the measurement device. Overall, both measurements are very similar to each other with a difference in the mean $R_{a}$ value of $\sim 3 \mathrm{~nm}$ showing the possibility of the optical device to perform roughness measurements that are comparable to traditional tactile devices.

In addition to profile based roughness measurements, the FV system is equipped with an area-based roughness module that allows a calculation of roughness measurements conform to a draft of an ISO standard on area-based roughness measurement [5].

In comparison to traditional profile based roughness measurement this allows a calculation of a much larger range of different surface texture parameters including amplitude parameters, volume parameters or the fractal dimension of the surface. The advantage of area based roughness measurement is that the results usually get more representative and repeatable due to a larger amount of data used for calculation. This module also allows the subtraction of different forms (spherical, cylindrical), two-dimensional Gaussian filtering and the filtration of measurement points with bad quality.

\subsection{Form Measurement of Hemi-Spherical Calottes}

Below, an evaluation of the proposed instrument with respect to form measurements is provided. The form of hemi-spherical calottes on
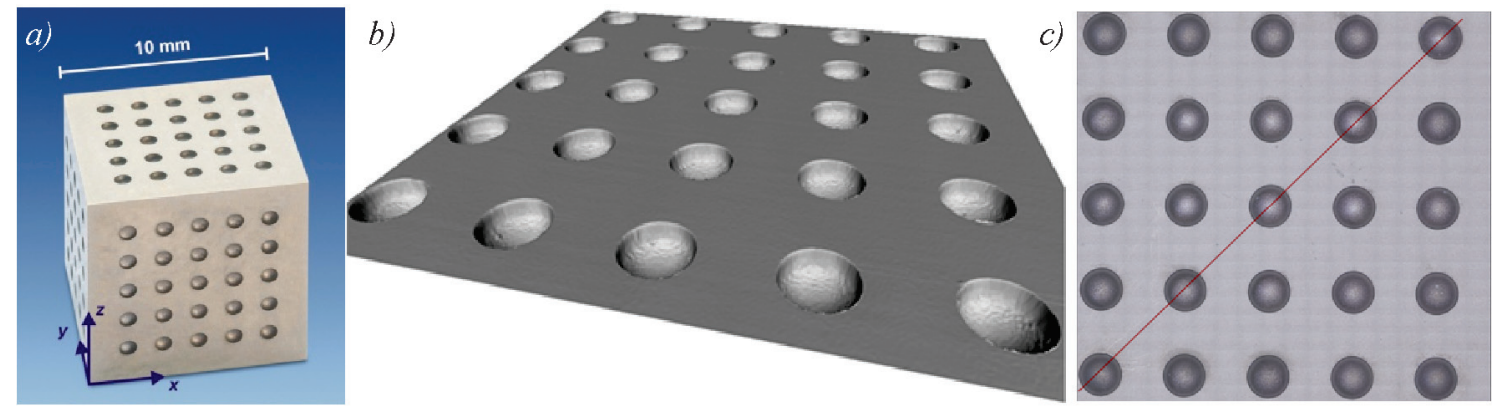

d)

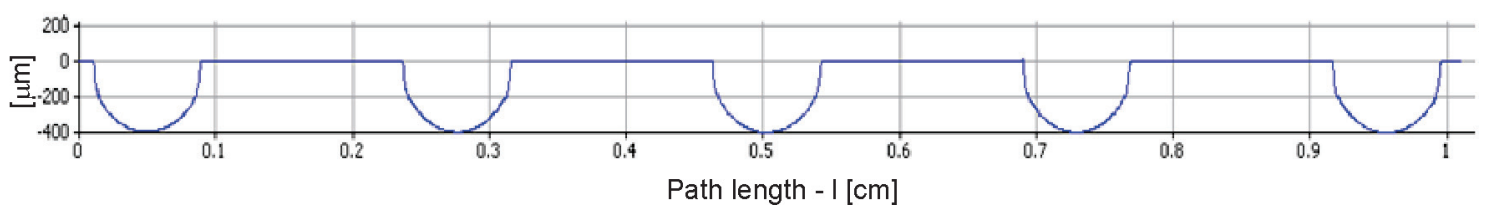

Fig. 5. Measurements on a calibration standard with hemi-spherical calotte, a) the PTB calibration standard, b) 3D dataset of one side of the calibration standard measured by focus variation, c) measured sharp true colour image with profile path and d) extracted height profile
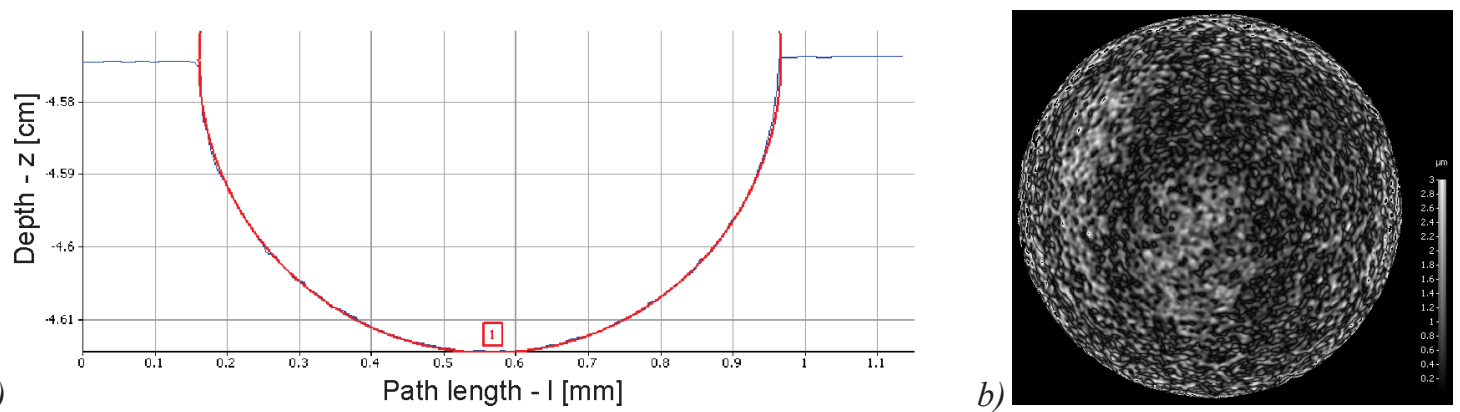

Fig. 6. Form measurements by focus variation, a) height profile of one calotte with fitted circle, b) absolute difference height map between measured and fitted sphere 
a calibration standard [16] developed by the PTB, the German national metrology institute has been measured. This calibration standard has the form of a cube with dimensions $10 \times 10 \times 10 \mathrm{~mm}$ with 25 hemi-spherical calottes with a nominal radius of $400 \mu \mathrm{m}$ on three faces (Fig. 5a).

Each side of the standard has a dimension of $10 \times 10 \mathrm{~mm}$, which can only be measured with devices that allow sufficiently large measurement areas. Secondly, the standard has to be measured with sufficient vertical resolution and accuracy to provide reliable data for the sphere fitting process. Thirdly, the device has to be able to measure even steep surface flanks since the calottes consist of surface patches with angles up to $90^{\circ}$. Below, measurements of this standard using the focus variation instrument described in Section 1.1. are provided In Fig. 5 the measurement results are demonstrated for one side of the calibration standard. In Fig. 5b a 3D dataset, which covers all 25 calottes on one side of the cube is shown. In Fig. 5c the measured sharp colour image is provided. Into this image a $2 \mathrm{D}$ profile path was drawn along which a surface profile was extracted as shown in Fig. 5d. Fig. 6a provides a detailed surface profile where a circle was fitted into the measured points. The fitted circle and the measured points show good correspondence even at the steep flanks. In Fig. $6 \mathrm{~b}$ a difference height dataset that shows the absolute differences between measured points and a sphere fitted in the least-squares sense, is provided. The deviations lie in a range between $0 \mu \mathrm{m}$ (dark grey-values) and 3 $\mu \mathrm{m}$ (bright grey-values).

In order to evaluate the repeatability of the system the radii of a sphere was measured 30 times in a row. The standard deviation sigma of the measurements is $\sim 15 \mathrm{~nm}$, which is rather small considering the sphere radius of $400 \mu \mathrm{m}$. The standard deviation can be converted into a confidence interval [mean $-2 \cdot \sigma$, mean $+2 \cdot \sigma]$ which is $\sim 60 \mathrm{~nm}$ and covers about $95 \%$ of all measurement results. The mean radius of the measurements is $402.594 \mu \mathrm{m}$. More information on the measurement of these samples can be found in [17].

\subsection{Wear Measurement of Cutting Tools}

In order to judge the quality of cutting tools, it is necessary to measure their geometry and wear during their use in the industrial process. This allows taking measures to improve the quality and durability of the tools as well as to increase the machining speed. Below, such measurements using the presented focus variation instrument are demonstrated.

The wear on corners of a milling cutter (Fig. 7) was measured. First 3D datasets of the corner (circle in Fig. 7) had been measured before and after usage. Afterwards, the difference between the two 3D datasets which contains

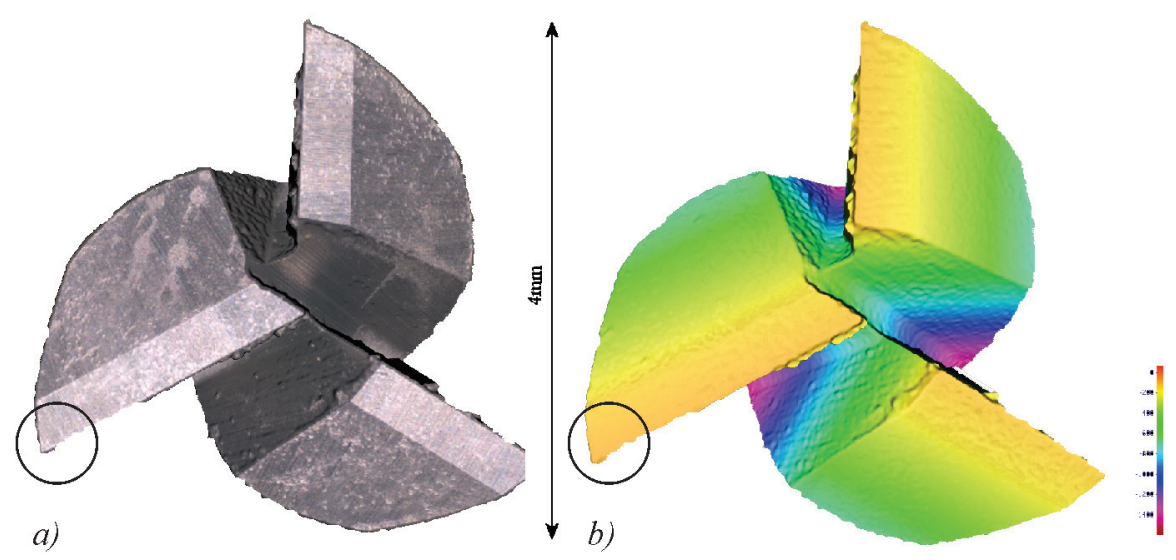

Fig. 7. 3D datasets of a milling cutter measured by focus variation; the parts that have been investigated in detail are marked with circles, a) 3D dataset with superimposed true color image, b) 3D dataset where each greyscale represents a different height 
the worn material, was calculated. In order to assure that the difference is calculated from corresponding surface regions, the two 3D datasets were registered to each other before difference calculation.

In Fig. $8 \mathrm{a}$ a $3 \mathrm{D}$ dataset of the original corner is provided, whereas Fig. 8b contains a $3 \mathrm{D}$ dataset of the used corner. Both 3D datasets were overlaid with the true color image measured by the focus variation instrument. This allows a classification into original regions (dark) and worn regions (bright). After the two 3D datasets were registered to each other, a difference height dataset was calculated (Fig. 8c) which allows the quantification of the worn volume $\left(\sim 601400 \mu \mathrm{m}^{3}\right)$. Another possibility to measure the amount of the worn volume is to extract height profiles of the original and the worn part and to overlay them (Fig. 8d) in a single diagram. This allows a good visualization of regions where much and regions where little material was removed. Additional measurements of milling cutters and cutting edges are provided by Danzl [18].

a)
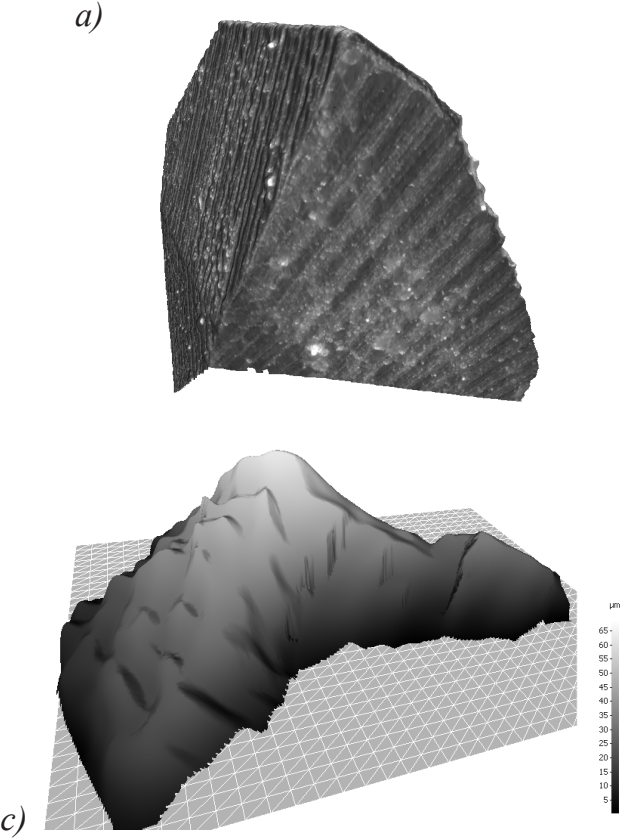

\subsection{Inspection of Welding Spots}

Laser beam welding is commonly used because of high-strength welding assembly and the small weld seam and the high qualitative weld seams without brittle occurrence. Checking and evaluating the classification of welding spots into good and bad parts during the production, saves expenses and time consuming rework. The combination of high resolution measurement data with the accordingly measured true color information is an important requirement for the classification. Due to this fact the localization and the topographic acquisition of high temperature oxidation can be realized.

Below, several results of welding spot measurements by focus variation are presented. There are many different criteria that are used for a classification into good and bad welding spots.

Typically, the discrimination of good and bad welding spots is performed by the following procedure:

1. Measure a 3D dataset of the welding spot.

2. Extract a surface profile of the welding spot. b)
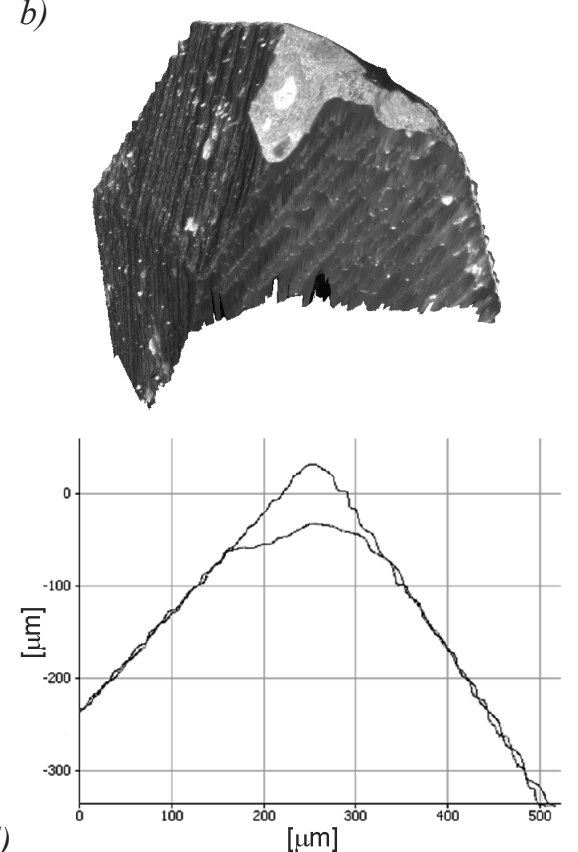

Fig. 8. Wear measurement of a corner of a milling cutter with focus variation, a) unused cutting edge, b) used cutting edge, c) difference volume of used and unused cutting edge, d) profiles of the used and unused cutting edge 
a)

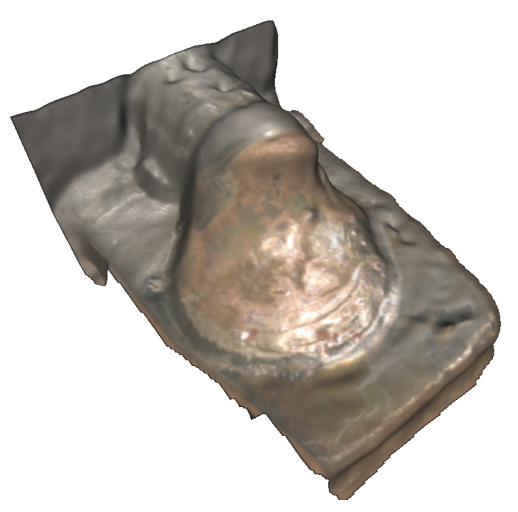

b)

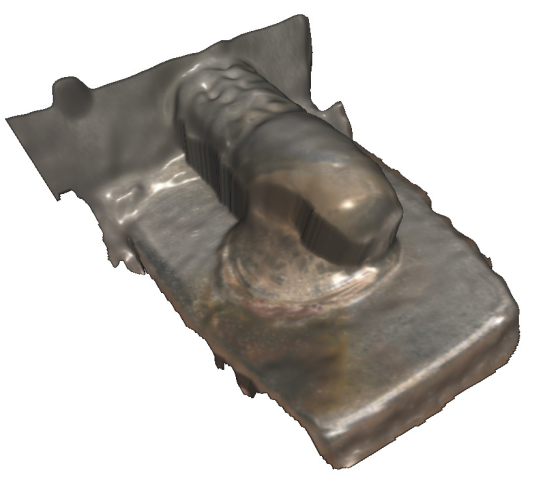

$\mathrm{mm}$
$-0.2-$
$-0.4-$
$-0.6-$
$-0.8-$
-1
$-1.2-$
-1.4
-1.6
-1.8
-2.
-2.2
-2.4

d)

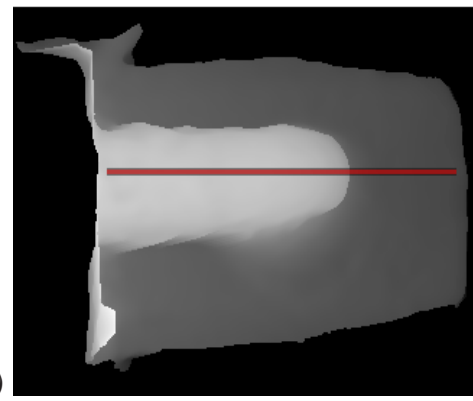

c)
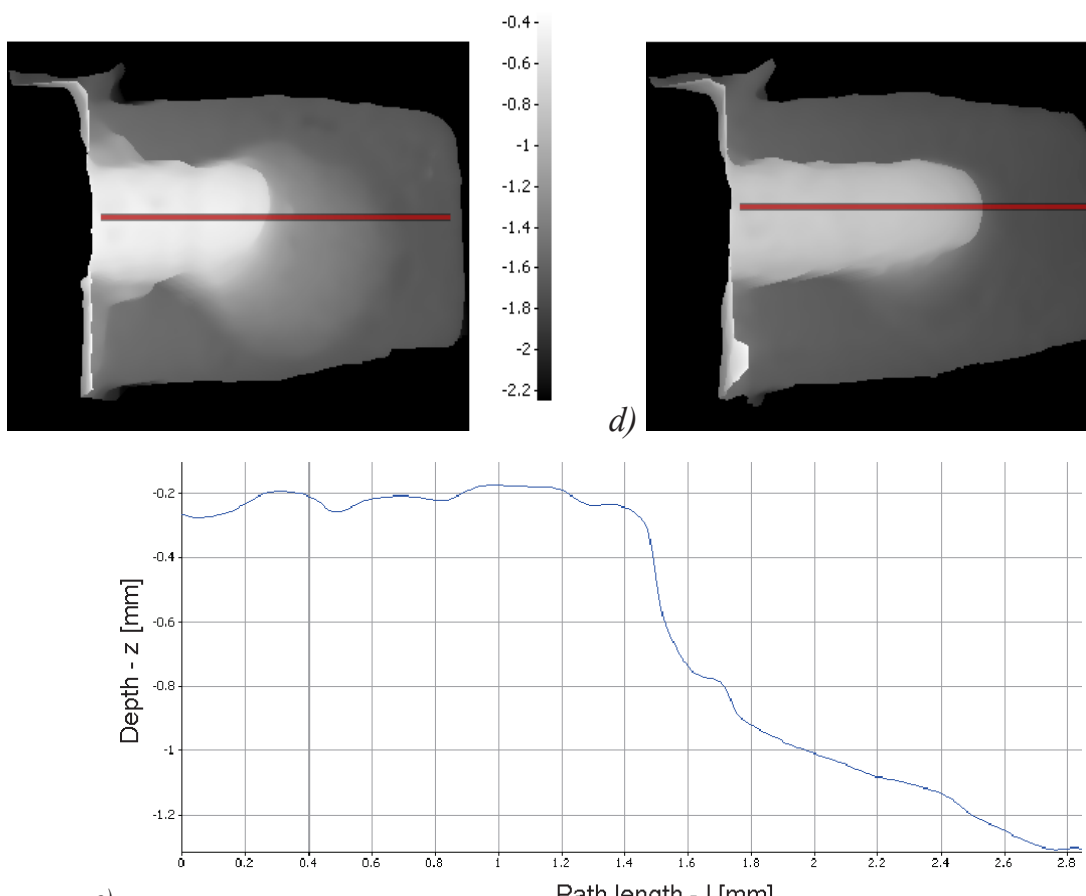

e)

Path length - I [mm]

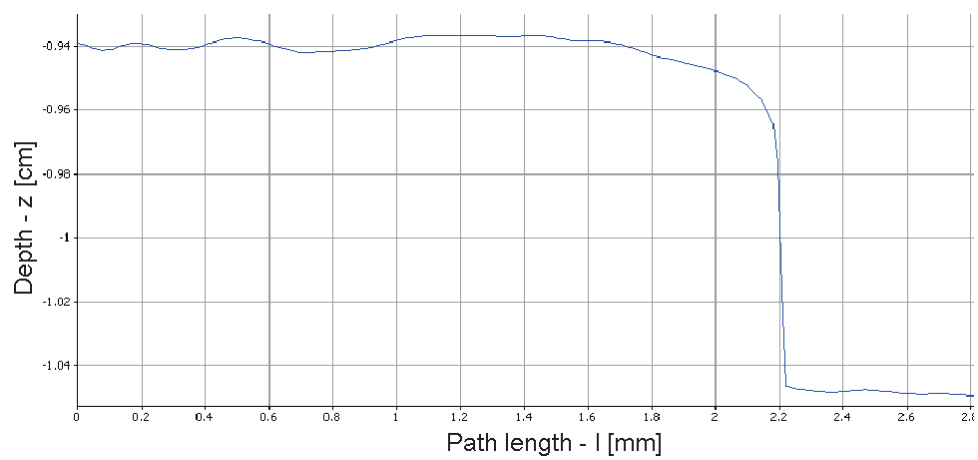

Fig. 9. Comparison of good $a-c)$ and bad welding spots $d-e), a-b)$ : visualization of the measured $3 D$ dataset, $c-d)$ : depth image with position of profile path, e-f) extracted profile; the bad welding spot has a very steep transition between the pin and the ground plate, whereas the good welding spot has a more smooth transition 
3. Extract discriminative parameters from the surface profile, the 3D dataset and the color information.

4. Decide on the quality of the welding spot on the basis of the extracted parameters.

The fourth step is typically performed by a classificator that has been trained by a series of labeled welding spots whose status (Ok, Not $\mathrm{Ok}$ ) is known. In Fig. 9 3D datasets of a good and bad welding spot are presented. The good welding spot in Fig. 9a has a smooth transition between the metal pin and the ground plate, whereas this transition is very abrupt for the bad welding spot in Fig. 9b. This is also visible in surface profiles in Figs. 9e and $f$ that were extracted along horizontal paths in Figs. 9c and d. A possibility for the discrimination of these two welding spots is to analyze the height histogram of the extracted surface profiles, the area and the position of the welding spot.

\section{CONCLUSIONS}

This article contains an evaluation of a focus variation instrument with respect to different measurement tasks including form, roughness and wear measurements. Roughness measurements on a newly developed roughness standard have provided very similar results of the focus variation instrument to traditional tactile devices. The $R_{a}$ differences are in the range of a few nanometers. Comparisons of measurements with traditional roughness standards show that the new standard is measurable much better due to its superimposed nano-roughness. Measurements on hemispherical calottes have shown an evaluation of the repeatability of the proposed system with respect to sphere measurements that were in the range of $<100 \mathrm{~nm}$. The evaluation of the system on two engineering applications shows that the system is able to measure steep surface flanks, which has been reported to be difficult for a series of other 3D measurement technologies [7]. This is the case for the milling cutter whose wear during the use in the industrial process could be quantified by means of $3 \mathrm{D}$ registration of $3 \mathrm{D}$ measurements. On the other hand, this is true for welding spot inspections which have a very irregular shape with steep flanks and difficult reflective behaviour.

\section{REFERENCES}

[1] Leach, R.K. (2009). Fundamental Principles of Engineering Nanometrology, William Andrew, Oxford.

[2] ISO 3274 (1996). Geometrical Product Specifications (GPS) - Surface texture: Profile method - Nominal characteristics of contact (stylus) instruments, International Organization of Standardization.

[3] ISO 5436-1 (2000). Geometrical Product Specifications (GPS) - Surface texture: Profile method; Measurement standards Part 1: Material measures, International Organization of Standardization.

[4] Jiang, X., Scott, P.J., Whitehouse, D.J., Blunt, L. (2007). Paradigm shifts in surface metrology; Part II; The current shift. Proceedings of the Royal Society, vol. 463, no. 2085, p. 2071-2099.

[5] ISO/DIS 25178-2. Geometrical product specifications (GPS) - Surface texture: Areal - Part 2: Terms, definitions and surface texture parameters. International Organization of Standardization.

[6] ISO 25178-6 (2010). Geometrical product specifications (GPS) - Surface texture: Areal - Part 6: Classification of methods for measuring surface texture. International Organization of Standardization.

[7] Vorburger, T.V., Rhee, H.G., Renegar, T.B., Song, J.F., Zheng, A. (2007). Comparison of optical and stylus methods for measurement of surface texture. International Journal of Advanced Manufacturing Technology, vol. 33, no. 1-2, p. 110-118.

[8] Gao, F., Leach, R.K., Petzing, J., Coupland, J.M. (2008). Surface Measurement errors using commercial scanning white light interferometers. Measurement Science and Technology, vol. 19, no. 1..

[9] Dietsch, M., König, N., Schmitt, R., Seewig, J. (2007). Sind taktile und optische Rauheitsmessungen vergleichbar?. VDI Berichte, vol. 1996, p. 187-206. (in German)

[10] Neugebauer, M., Neuschaefer-Rube, U. (2005). A new micro artefact for testing of optical and tactile sensors. Proc. of the $5^{\text {th }}$ Euspen International Conference and $7^{\text {th }}$ annual general meeting of the European 
society for precision engineering and nanotechnology, Montpellier, p. 201-204.

[11] Danzl, R., Helmli, F., Scherer, S. (2006). Comparison of roughness measurements between a contact stylus instrument and an optical measurement device based on a colour focus sensor. Proc. of the Nanotechnology Conference, Boston, vol. 3, p. 284-287.

[12] Ren, Y.-F., Zhao, Q., Malmstrom, H., Barnes, V., Xu, T. (2009). Assessing fluoride treatment and resistance of dental enamel to soft drink erosion in vitro: applications of focus variation 3D scanning microscopy and stylus profilometry. Journal of Dentistry, vol. 37, no. 3, p. 167-176.

[13] Lechthaler, M., Bauer, W. (2006). Rauigkeit und Topografie - ein Vergleich unterschiedlicher Messverfahren. Wochenblatt für Papierfabrikation, vol. 21, no. 21, p. 1227-1234

[14] Scherer, S. (2007). Focus-Variation for optical 3D measurement in the micro- and nano-range. Bauer, N. (ed.) Handbuch zur
Industriellen Bildverarbeitung, Fraunhofer IRB Verlag, p. 198-210.

[15] Rubert, P. (2008). Report on the results of an intercomparison, using stylus-type and non-contacting surface measurement instruments. Proc. XII. Int. Colloquium on Surfaces, Chemnitz.

[16] Neugebauer, M., Hilpert, U., Bartscher, M., Gerwien, N., Kunz, S., Neumann, F., Goebbels, J., Weidemann, G. (2007). Ein geometrisches Normal zur Prüfung von Röntgen-Mikrocomputertomographiemess systemen. Technisches Messen, vol. 74, no. 11, p. 565-571. (In German)

[17] Danzl, R., Helmli, F., Scherer, S. (2007). Automatic measurement of calibration standards with arrays of hemi-spherical calottes. Proc. 11th Int. Conf. on Metrology and Properties of Engineering Surfaces, Huddersfield, p. 41-46.

[18] Danzl, R., Helmli, F. (2008). Geometry and wear measurement of cutting tools. Int. Conf. on High Performance Cutting, Dublin, vol. 1, p. 111-118. 